\title{
natureINSIGHT
}

\section{TRANSCRIPTION AND EPIGENETICS}

24 October 2013 / Vol 502 / Issue No 7472

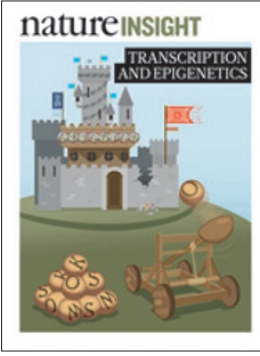

Cover illustration Illustration by Nik Spencer (concept: Effie Apostolou and John Bardakos)

\section{Editor, Nature}

Philip Campbell

Publishing

Richard Hughes

Production Editor Jenny Rooke

Art Editor

Nik Spencer

Sponsorship

Reya Silao

Production

lan Pope

Marketing

Elena Woodstock

Steven Hurst

Editorial Assistant

Abbie Williams

Anastasia Panoutsou $\eta$ ranscriptional programs drive cell identity, dictating the specialized phenotypes of differentiated cells during the development of multicellular organisms. Interplay between signaltransduction pathways, transcription factors and the chromatin packaging of the genome sets the gene expression pattern of a cell, which must be relatively stably maintained once an organism is fully developed. The Reviews in this Insight explore how transcriptional states are regulated during development and disease.

The discovery that differentiated cells can be artificially reprogrammed into induced pluripotent stem cells by a small set of transcription factors has opened up exciting medical prospects and provided an opportunity to investigate how stable epigenetic states are built and reversed. Effie Apostolou and Konrad Hochedlinger discuss transcriptional and chromatin-based mechanisms behind cellular reprogramming and draw comparisons with tumorigenesis.

DNA methylation is a relatively stable epigenetic mark that locks genes in a silenced state, but pathways involved in removing DNA methylation marks are now becoming clear. Mechanistic models for these demethylation pathways are presented by Rahul Kohli and Yi Zhang, who highlight a key role for TET enzymes in various biological processes.

Transcriptional states become perturbed in disease, and genes encoding chromatin-associated proteins are often aberrantly expressed or mutated in cancer. Kristian Helin and Dashyant Dhanak discuss the latest discoveries of specific chemical inhibitors directed against chromatin regulators, as clinical trials of these compounds begin.

The transcriptional program of differentiated cells retains plasticity to ensure appropriate responses to the cellular environment. Philipp Gut and Eric Verdin propose that the metabolic state of a cell can directly influence DNA and histone modifications and thus help to regulate gene expression.

Finally, Wouter de Laat and Denis Duboule discuss the DNA regulatory elements termed enhancers that are at the heart of developmental transcription regulation and ensure the correct spatio-temporal expression of genes. Recent insight into the three-dimensional topology of chromosomes adds to our understanding of how enhancers act through long-range regulatory contacts.

Alex Eccleston, Francesca Cesari \& Magdalena Skipper Senior Editors

\section{CONTENTS}

\section{REVIEWS}

462 Chromatin dynamics during cellular reprogramming Effie Apostolou \& Konrad Hochedlinger

472 TET enzymes, TDG and the dynamics of DNA demethylation Rahul M. Kohli \& Yi Zhang

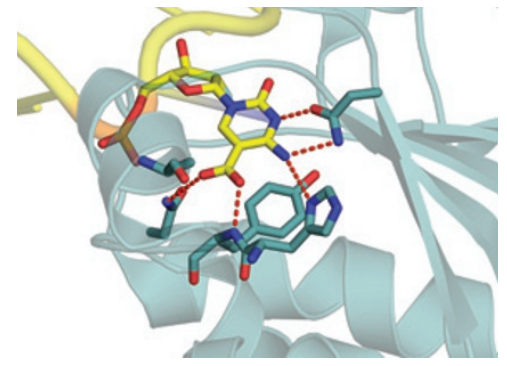

480 Chromatin proteins and modifications as drug targets

Kristian Helin \& Dashyant Dhanak

489 The nexus of chromatin regulation and intermediary metabolism Philipp Gut \& Eric Verdin

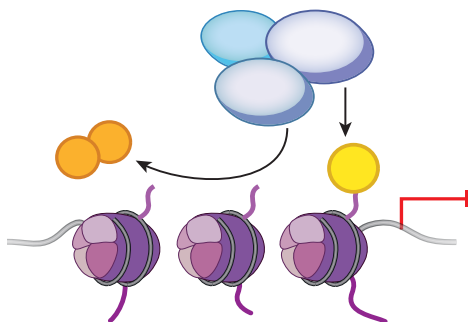

499 Topology of mammalian developmental enhancers and their regulatory landscapes

Wouter de Laat \& Denis Duboule

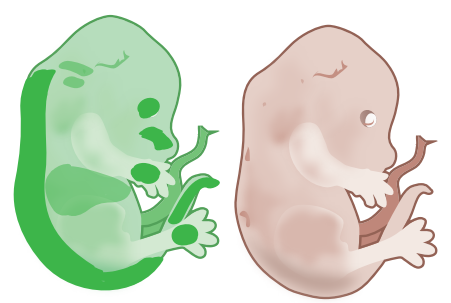

